\title{
The Effects of Combined Training on Oxidative Stress and Antioxidant Defense Indicators
}

\author{
Reza Delavar ${ }^{*}$, Mahdi Mogharnasi², Nazanin Khoobkhahi ${ }^{3}$ \\ ${ }^{1}$ Assistant Professor, Department of Sport Sciences, Faculty of Education and Psychology, University of Sistan and Baluchestan, \\ Zahedan, Iran \\ ${ }^{2}$ Associate Professor, Department of Sports Physiology, Faculty of Sport Sciences, University of Birjand, Birjand, Iran \\ ${ }^{3} \mathrm{MSc}$ Student, Department of Sport Sciences, Faculty of Education and Psychology, University of Sistan and Baluchestan, \\ Zahedan, Iran
}

\section{*Correspondence to}

Reza Delavar, Assistant Professor, Department of Sport Sciences, Faculty of Education and Psychology, University of Sistan and Baluchestan, Zahedan, Iran. Tel: +985431132674; Email: delavar@ped.usb.ac.ir

Received February 1, 2017 Accepted March 6, 2017 Published online March 18, 2017

\begin{abstract}
Introduction: Physical activity and exercise due to increased cellular oxidative process lead to increased free-radical production and reactive oxygen species. "Oxidative stress" is defined as the disturbed balance between oxidative and antioxidative indicators in favor of oxidative species.

Methods: In this semi-experimental study, 23 sedentary boys volunteered to participate. They were randomly assigned to 2 groups of training $(n=13)$ and control $(n=10)$. The combined trainings included endurance running with $60 \%$ to $80 \%$ of maximum heart rate and resistance weight training with $40 \%$ to $50 \%$ and one repetition maximum intensity. Blood samples were taken from the subjects' forearm vein, 24 hours before and 48 hours after the last training session in a fasting state. Paired and independent $t$ tests were used to locate the inner-group and inter-group changes respectively. Data were analyzed using SPSS version 20. A $P<0.01$ was considered significant.

Results: Malondialdehyde (MDA) level as a marker of oxidative stress in the exercise group decreased significantly after 24 sessions of exercise $(P=0.015)$, while the index of total antioxidant capacity (TAC) significantly increased in the exercise group $(P=0.021)$. There was no significant difference in the 2 indexes in the control group $(P<0.05)$.

Conclusion: The present study suggests that combined training provides a good balance between indicators of oxidative stress and antioxidant defense among sedentary boys and this would prevent the overproduction of free radicals.

Keywords: Combined training, Oxidative stress markers, Antioxidant defense.
\end{abstract}

Please cite this article as follows: Delavar R, Mogharnasi M, Khoobkhahi N. The effects of combined training on oxidative stress and antioxidant defense indicators. Int J Basic Sci Med. 2017;2(1):29-

32. doi: $10.15171 /$ ijbms.2017.07.

\section{Introduction}

Sports science is a new discipline that has been introduced to the world in recent years through the works of scientists and researchers interested in this realm. This discipline is growing in demand due to its different uses and a variety of applications, and almost all the people and social institutions are getting involved with this field of study. ${ }^{1}$ The state of imbalance between generation of reactive free radicals and the antioxidative capacities detoxifying their detrimental effects is called oxidative stress. The shift in the balance between oxidants and antioxidants in favor of oxidants is termed "oxidative stress." 2 Stress oxidative can damage tissues, cells and macromolecule of cells like lipids,
\end{abstract}

proteins and nucleic acids. Thus, oxidative stress leads to many conditions in the body like reduction in physical performance, chronic fatigue, tissue damage and over training. ${ }^{3}$ Antioxidants are molecules that protect biological systems against chain reactions that lead to oxidation. ${ }^{4}$

A variety of studies have focused on exercises with different protocols on antioxidant and oxidative stress indicators. Firoozrai et al found that cell membrane of red blood cells and maybe other body cells is less susceptible to damage among athletes indicating that the balance between antioxidants and oxidative stress is more suitable among athletes than among other people. ${ }^{5}$ Gaeini et al showed that after one session of endurance

\footnotetext{
Copyright (C) 2017 The Author(s); Published by Zabol University of Medical Sciences. This is an open-access article distributed under the terms of the Creative Commons Attribution License (http://creativecommons.org/licenses/by/4.0), which permits unrestricted use, distribution, and reproduction in any medium, provided the original work is properly cited.
} 
training malondialdehyde (MDA) reduced compared with control group. ${ }^{6}$ Dantas et al showed a reduction in MDA and an increase in total antioxidant capacity (TAC) after strength training among elderly women. ${ }^{7}$ In another study Pedersen et al indicated that daily taking of methylsulfonylmethane supplement for 10 days can reduce muscle damage by affecting TAC in healthy men. ${ }^{8}$ The biological effects of oxidizing compounds in the body are controlled by antioxidant factors. Dysfunction of the organs might be due to reactions of free radicals with cell membrane. It is known that the main aim of oxygen radicals is the lipids in cell membrane. Human body tissues just like Erythrocytes contain important antioxidants such as glutathione peroxidase and glutathione, therefore normal erythrocytes can resist better oxidative damages. ${ }^{9}$ Due to lack of relevant literature related to the effect of combined training on the above-mentioned indicators, in this research we intend to analyze the effect of combined training (resistance and endurance) and the interaction between oxidative stress and antioxidant defense. In addition, we aim to find the answer to the following questions:

1. Does an 8-week combined training (resistance and endurance) have a significant effect on oxidative stress and antioxidant defense indicators among sedentary boys?

2. Do we see a significant change due to training compared to the control group?

\section{Methods}

In this quasi-experimental study the statistical population included all the boys in Zahedan Youth Detention Center with the age range between 15 to 25 years old. From this population, 23 boys volunteered to participate in the research and were divided randomly into 2 groups (13 in the training group and 10 in the control group). Subjects were first introduced to the aims and process of the research then they were asked to fill out and sign a consent form. In order to avoid any interrupting factors that might affect the study outcomes, subjects were checked regarding their well-being before research initiation. In addition, medication use and suffering from a special disease were taken into account. By the same token a medical health questionnaire was filled by the doctor for each participant. Subjects in the control group were asked not to take part in any physical activity during the time the protocol was being experimented. ${ }^{10}$

\section{Combine Training Protocol}

The training program was performed 3 times per week for 8 weeks. The combined training included resistance and endurance exercises in a way that a training session started with a 10-minute general warm-up, then the resistance and endurance exercises and a 5-minute cool down at the end of each session. ${ }^{11}$

\section{Endurance Training Protocol}

The endurance training program in the first 2 weeks
Table 1. Endurance Training Protocol

\begin{tabular}{lccc}
\hline $\begin{array}{l}\text { Session } \\
\text { Number }\end{array}$ & Week & $\begin{array}{c}\text { Training Time } \\
(\mathbf{m i n})\end{array}$ & $\begin{array}{c}\text { Training Intensity } \\
\mathbf{( \% )}\end{array}$ \\
\hline 1 & First & 15 & $60-65$ \\
2 & Second & 15 & $60-65$ \\
3 & Third & 20 & $65-70$ \\
4 & Fourth & 20 & $65-70$ \\
5 & Fifth & 25 & $70-75$ \\
6 & Sixth & 25 & $70-75$ \\
7 & Seventh & 30 & $75-80$ \\
8 & Eighth & 30 & $75-80$ \\
\hline
\end{tabular}

included running for 15 minutes with $60 \%$ to $65 \%$ of maximum heart rate that increased into 30 minutes in the eighth week with $75 \%$ to $80 \%$ of maximum heart rate. Therefore, 5 minutes were added to the training time in each 2 weeks and the intensity of the training increased $5 \%$ (see Table 1$)^{12}$

\section{Resistance Training Protocol}

The resistance training program included bench press, legs, shoulders, squat and armpit which were done in a circular way. In the first week of training, exercises with $40 \%$ to $50 \%$ one repetition maximum (1RM) were performed then they increased to $50 \%$ to $55 \%, 55 \%$ to $60 \%, 60 \%$ to $65 \%, 65 \%$ to $70 \%, 70 \%$ to $75 \%, 75 \%$ to $80 \%$ and $80 \%$ to $85 \% 1 \mathrm{RM}$ for second, third, fourth, fifth, sixth, seventh and eighth weeks, respectively. ${ }^{13}$

\section{Blood Biochemical Measurements}

Blood samples were taken after 8-10 hours of fasting between $7 \mathrm{AM}$ to $9 \mathrm{AM}$ in the morning in 2 stages; one day before the beginning of the training and 48 hours after the last training session. Blood was taken from the right arm of each participant while sitting in a relaxed manner. About $10 \mathrm{~mL}$ of blood was taken and the sera were separated immediately by using centrifugation with the speed of $3000 \mathrm{rpm}$ for 5 minutes. The blood samples were kept at $-70^{\circ} \mathrm{C}$ for further analysis. The intended indicators (MDA and TAC) were measured in a specialized laboratory by using ELISA method and Human Malondialdehyde ELISA kit made in China-USA with sensitivity of 0.22 $\mathrm{nmol} / \mathrm{ml}$ and Human Total Antioxidant kit made in China-USA with sensitivity of $0.03 \mathrm{unit} / \mathrm{mL}$.

\section{Statistical Analysis}

Kolmogorov-Smirnov test was used to confirm that the data were natural. To analyze inner group changes of variables paired $t$ test was used and to analyze inter group variables an independent $t$ test was applied. Data were analyzed by SPSS version 20 with a significance level at $P<0.05$.

\section{Results}

Findings showed that the amount of MDA decreased and the amount of TAC increased in the experimental group. Table 2 shows the mean and standard deviation of 
Table 2. The Mean and Standard Deviation Values of the Studied Variables in Experimental and Control Groups

\begin{tabular}{|c|c|c|c|c|c|}
\hline \multirow{2}{*}{ Groups } & \multirow{2}{*}{ Variables } & \multicolumn{2}{|c|}{ Measurement Time } & \multirow{2}{*}{ Inner-group $P$} & \multirow{2}{*}{ Inter-group $P$} \\
\hline & & Pre-test & Post-test & & \\
\hline Training & \multirow{2}{*}{ MDA $(\mathrm{nmol} / \mathrm{mL})$} & 8.43 & 7.75 & $0.01^{\mathrm{a}}$ & \multirow{2}{*}{$0.001^{a}$} \\
\hline Control & & 8.06 & 8.41 & 0.35 & \\
\hline Training & \multirow{2}{*}{$\mathrm{TAC}(\mu / \mathrm{mL})$} & 11.34 & 13.27 & $0.02^{\mathrm{a}}$ & \multirow{2}{*}{$0.03^{a}$} \\
\hline Control & & 11.12 & 11.06 & 0.90 & \\
\hline
\end{tabular}

Abbreviation: MDA, malondialdehyde; TAC, total antioxidant capacity.

a Significant at $P<0.05$.

variables. The average amount of MDA and TAC in control group did not change significantly when comparing pretest and post-test results. In order to analyze the difference in the amount of MDA and antioxidant capacity between the control and training group, independent $t$ test was used and the results of this test showed that the difference between the 2 groups was significant.

\section{Discussion}

The results of the current study with respect to TAC and MDA indicate that MDA amount after 8 weeks of combined training decreases significantly and the amount of TAC increases as well.

Concerning TAC, our findings are in line with the results of previously conducted studies. Farzanegi et al described that levels of TAC increased while MDA decreased following 6 weeks of aerobic training in postmenopausal women affected with hypertension. ${ }^{14}$ Jamurts et al also observed an increase in TAC marker in all training groups and a decrease in MDA just in the endurance training group due to resistance and endurance exercises which were held 3 times per week for 6 weeks among the sedentary elderly. ${ }^{15}$ In addition, long-term sports activity in a study by Padayatty et al demonstrated an increase in TAC which caused an imbalance in pro-oxidants and antioxidants. ${ }^{16}$ In another study an increase in the activity of antioxidant enzymes and a decrease in the level of MDA in hippocampus of diabetic male rats had beneficial effects in preventing neurological complications of diabetes mellitus and tissue damage due to oxidative stress caused by this disease. ${ }^{17}$ In a study by Fakoori Jouybari et al a decrease in MDA in relaxed levels and an increase of TAC in all three experiment groups due to 8 weeks of aerobic exercise with purslane seeds consumption on pro-oxidant and antioxidants indicators among women with type 2 diabetes were observed. ${ }^{18}$ On the other hand, Firoozrai et al analyzed the effects of sports on the reduction of cell membrane susceptibility in antioxidant defense and oxidative stress conditions for 6 months and did not find a significant difference in TAC which is not consistent with the results of our study. ${ }^{5}$ Probably, existence of resistance training in this research and its combination with endurance training had more compatibility with this indicator, while in the study of Firoozrai et al due to lack of resistance training such a compatibility was not achieved.
Also, Marzatico et al found that MDA levels in plasma increased 48 hours after fast activities among fast athletes and immediately after endurance activity in marathon runners. ${ }^{19}$ This finding is not in line with the results of the present research. We can conclude that this increase is on account of shorter period of time in training protocol or because of existence of a fast training protocol in the above-mentioned research. In a study by Park et al which analyzed the impact of aerobic and anaerobic exercise training on oxidative stress and antioxidant defense in athletes, MDA increased and TAC decreased which is inconsistent with our results. This difference can be due to different levels of physical readiness in the studies as well as subjects which were athletes. ${ }^{20} \mathrm{~A}$ variety of mechanisms in explaining the TAC reaction to exercise shows that after intense sport trainings an increase in oxidative stress is caused but combined trainings decrease production of free radicals if done continuously. ${ }^{21}$ Therefore, MDA decreases as an indicator of lipid pro-oxidation. After the reduction of oxidative stress in body, antioxidant defense system in order to balance with combining resistance and endurance training in a several months' period activates and this increase needs consumption of anti-oxidant supplements to have more effects. ${ }^{21}$ The most important result of this study is the effectiveness of combined training which has a positive effect on oxidative stress and antioxidant defense indicators by decreasing MDA and increasing TAC.

\section{Conclusion}

According to the findings of this research, combined training (resistance-enduring), causes a reduction in oxidative stress markers such as MDA. The reduction of this indicator is accompanied with an increase in TAC. Thus, such trainings to improve the anti-oxidation capacity are suggested. However, to understand the effects of these trainings in different times and combined with anti-oxidation supplements, doing more research will be helpful.

\section{Ethical Approval}

This study has been reviewed by the Research Ethics Committee of Sport Sciences Research Institute and was approved according to compliance with Ethical Standards in Research of the Ministry of Science, Research and 
Technology, with the Code IR.SSRI.REC.1395.123.

\section{Competing Interests}

Authors declare that they have no competing interests.

\section{References}

1. Arazi H, Rahmaninia F, Azali K, Mehrtash M. The effect of acute L-carnitine supplementation on the blood lactate, glucose, $\mathrm{VO} 2 \mathrm{max}$ and power in trained men. Tehran Uni Med J. 2003;71(1): 59-64.

2. Choi SW, Benzie IF, Collins AR, Hannigan BM, Strain JJ. Vitamin C and E acute interactive effects on biomarkers of antioxidant defence and Oxidative stress. Mutat Res. 2004;551(1-2):109-117. doi: 10.1016/j. mrfmmm.2004.03.006.

3. Constantin-Teodosiu D, Howell S, Greenhaff PL. Carnitine metabolism in human muscle fiber types during submaximal dynamic exercise. J Appl Physiol (1985). 1996;80(3):1061-1064.

4. Han D, Loukianoff S, Mclaughlin L, et al. Handbook of Oxidants and Antioxidants in Exercise. Amsterdam: Elsevier; 2000:433-484.

5. Firoozrai M, Sarasgani M, Hesabi B, Bandegi A. Effect of sports on the reduction of cell membrane susceptibility, antioxidant defense and oxidative stress. Razi J Med Sci. 2006;14(56):125-136.

6. Gaeini AA, Sheykh Al Eslami Vatani D, Alameh AA, et al. Effect of endurance ttraining and detraining on lipid peroxidation and antioxidant system Wistar rats. Journal of Movement Science \& Sports. 2008;6(11):51-63.

7. Dantas FF, Brasileiro-Santos Mdo S, Batista RM, et al. Effect of strength ttraining on oxidative stress and the correlation of the same with forearm vasodilatation and blood pressure of hypertensive elderly women: a randomized clinical trial. PLoS One. 2016;11(8):e0161178. doi:10.1371/journal. pone.0161178.

8. Pedersen BK, Saltin B. Evidence for prescribing exercise as therapy in chronic disease. Scand J Med Sci Sports. 2006; (Suppl 1) PP. 3-36. doi:10.1111/j.1600-0838.2006.00520.x

9. Salehi I, Mohammadi M, Farajnia S, et al. Effect of regular swimming on oxidative stress and atherogenic index in blood of diabetic male rats (Persian). Scientific Journal of Hamadan University of Medical Sciences. 2007;14(3):2935.

10. Galdavi R, Mogharnasi M. The effect of two methods of endurance and resistance training on omentin-1 levels of plasma and factors related to obesity in overweight and obese girls in university of Sistan and Baluchestan (Persian). Iranian Journal of Diabetes and Metabolism.
2016;15(2):101-109.

11. Abedi B, Abbasi-Bakhtiari R. The effect of a 12-week combined training program on serum leptin, C- reactive protein and the insulin resistance index in overweight men (Persian). Feyz. 2015; 19(4): 293-301.

12. Gorzi A, Rajabi H, Azad A, et al. Effect of concurrent, strength and endurance training on hormones, lipids and inflammatory characteristics of untrained men (Persian). Iranian Journal of Endocrinology and Metabolism. 2012;13(6):614-620.

13. Saremi A, Gheraati MR. The effect of resistance training on serum myostatin level and insulin resistance in obeseoverweight men (Persian). Sport Biosciences. 2010; 2(4):93-108.

14. Farzanegi P, Habibian M, Kaftari A. Effect of 6-weeks aerobic exercise training on oxidative stress and enzymatic antioxidants in postmenopausal women with hypertension: case study (Persian). J Mazandaran Univ Med Sci. 2014;23(108): 134-136.

15. Jamurts AZ, Fatouros IG, Deliconstantinos G, et al. Chronic endurance and resistance exercise effects on oxidative stress and antioxidant status of inactive older adults. Med Sci Sport Exer. 2003;35(5): 78- 94. doi:10.1097/00005768200305001-00533.

16. Padayatty SJ, Katz A, Wang Y, et al. Vitamin C as an antioxidant: evaluation of its role in disease prevention. J Am Coll Nutr. 2003;22(1):18-35.

17. Mohammadi M, Salehi I, Farajnia SA. Effect of swimming exercise on oxidative stress in hippocampus of diabetic male rats. Medical Journal of Tabriz University of Medical Scineces. 2008;30( 2):111-118.

18. Fakoori Jouybari M, Farzanegi Arkhazlue P, Hashemvarzi A. The effect of 8 weeks aerobic exercise with Purslane seeds consumption on peroxidant and antioxidants indicators in women with type 2 diabetes. Int J Sport Stud. 2014;4(2):238-245.

19. Marzatico F, Pansarasa O, Bertorelli L, Somenzini L, Della Valle G. Blood free radical antioxidant enzymes and lipid peroxides following long-distance and lactacidemic performances in highly trained aerobic and sprint athletes. J Sports Med Phys Fitness. 1997;37(4):235-239.

20. Park SY, Kwak YS. Impact of aerobic and anaerobic exercise training on oxidative stress and antioxidant defense in athletes. J Exerc Rehabil. 2016;12(2):113-118. doi:10.12965/ jer.1632598.299.

21. Rahbar S, Ahmadiasl N. Effect of long term regular resistance exercise on heart function and oxidative stress in rats. Journal of Ardabil University of Medical. 2012;12(3);256-264. 